\title{
Memory lost, memory regained: neuropsychological findings and neuroimaging in two cases of paraneoplastic limbic encephalitis with radically different outcomes
}

\author{
T H Bak, N Antoun, K K Balan, J R Hodges
}

\begin{abstract}
Objective-To report two cases of paraneoplastic limbic encephalitis (PNLE) with similar clinical presentation, but dramatically different outcome and to highlight the role of neuropsychological and radiological evaluation in PNLE.
\end{abstract}

Methods-Both patients underwent an extensive battery of neuropsychological tests designed to document general intellectual function, anterograde verbal and visual memory, naming, knowledge and executive ability. In addition, structural (CT and MRI) and functional (HMPAOSPECT) brain scans were performed. Results-Both patients presented with fairly sudden onset of profound and persistent memory loss in the absence of other neurological symptoms. Their subsequently diagnosed small cell lung cancer was treated with a combination of radiotherapy and chemotherapy, leading to remission of the tumour. The memory of patient 1 recovered fully and he died from an unrelated cause 1 year later; neuropsychological testing showed a severe, but isolated, anterograde amnesia, brain MRI was normal and HMPAO-SPECT showed left medial temporal hypoperfusion. Patient 2 remained densely amnesic despite regression of her lung tumour; neuropsychological testing disclosed both anterograde and extensive retrograde amnesia together with more generalised cognitive deficits including anomia and executive impairments, MRI showed gross atrophy of the hippocampus and amygdala bilaterally, and HMPAO-SPECT showed pronounced frontal and temporal hypoperfusion.

Conclusion-Complete remission from PNLE may occur and seems to be associated with pure anterograde amnesia without evidence of structural hippocampal damage in MRI. By contrast, cognitive deficits beyond severe anterograde amnesia and evidence of destructive medial temporal lobe pathology on MRI seem to be poor prognostic features.

(F Neurol Neurosurg Psychiatry 2001;71:40-47)

Keywords: paraneoplastic limbic encephalitis; amnesia; semantic memory

Professor J R Hodges

john.hodges@

mrc-cbu.cam.ac.uk

Received 6 April 2000 and in revised form

13 November 2000

Accepted 12 March 2001
Subacute limbic encephalitis was first described as a separate syndrome by Brierly et al in $1960 .{ }^{1}$ Its association with neoplasms was postulated a few years later by Corellis et al. ${ }^{2}$ Since then paraneoplastic limbic encephalitis (PNLE) has been recognised as a rare, non-metastatic complication of malignant tumours. It can occur either alone or in combination with other paraneoplastic syndromes - for example, paraneoplastic cerebellar degeneration. ${ }^{3}{ }^{4}$ The symptoms can develop either suddenly ${ }^{5}$ or in a slowly progressive way. ${ }^{6}$ In $75 \%$ of cases the primary tumour is a small cell carcinoma of the lung, but an association with many other tumours including thymoma, lymphoma, colon adenocarcinoma, or testicular tumours has been reported. ${ }^{7}$ Paraneoplastic limbic encephalitis may precede the diagnosis of the underlying tumour by several months. The principal symptom is severe amnesia, either in isolation or combined with other cognitive deficits, epileptic seizures, or psychiatric symptoms including hallucinations, confusion, and agitation. Examination of CSF often shows abnormal cells or increased protein. Neuroimaging, particularly MRI, can show medial temporal hyperintensity or atrophy, ${ }^{8-11}$ although involvement of other areas - for example, insula and basal gangliahas also been reported. ${ }^{7}$ FDG-PET scanning may show focal hyperactivity involving the medial temporal lobes. ${ }^{12}{ }^{13}$ The presence of anti-Hu antibodies in nervous tissue, serum, and CSF is often reported, ${ }^{14}$ but their absence does not exclude the diagnosis: many histologically confirmed cases show negative antibody results. ${ }^{15} 16$

The course of PNLE is usually rapidly progressive over weeks to months. Occasional cases of spontaneous remission ${ }^{17}$ and improvement due to antineoplastic treatment ${ }^{18-20}$ have both been reported, but the neuropsychological documentation of recovery is virtually absent. As yet, no clear prognostic factors have been established, although it has been suggested that the likelihood of recovery corresponds to the absence of antineuronal antibodies. ${ }^{15}$ Although it is generally agreed that an amnestic syndrome forms the core of PNLE, comparatively little is known about the exact nature of the memory impairment or the possible involvement of other cognitive domains. Few authors have used standardised neuropsychological tests to differentiate between the different components of memory and many studies have been complicated by concurrent epileptic seizures and anticonvulsant medication. $^{2122}$ 
The main aim of the present study was to document the neuropsychological and neuroimaging findings in two contrasting patients with limbic encephalitis, one of whom recovered completely after chemotherapy for small cell bronchial carcinoma. Secondary aims were to report (1) the dissociation between abnormal functional (SPECT) and normal structural (MRI) imaging in the patient with full recovery; (2) the finding of profound bilateral hippocampal and amygdalar atrophy in the patient with poor outcome (case 2); and (3) the relation between anterograde and remote memory: severe anterograde without retrograde amnesia was seen in patient 1 whose pathology was apparently limited to the left temporal lobe versus patient 2 who had marked retrograde amnesia in association with more widespread cerebral involvement.

\section{Case 1}

PRESENTATION

A 65 year old right handed male university graduate currently working as an insurance executive (with international expertise in damage estimation after natural disasters) was referred in December 1995 to Addenbrooke's Hospital, Cambridge because of a sudden and persistent loss of memory with no other accompanying symptoms. The clinical picture resembled transient global amnesia ${ }^{2324}$ notably severe disorientation, loss of recollection for recent events, and repetitive questioning. By contrast, remote memory remained unaffected: he could recall events accurately from the past, including details of his stay in America 2 years before presentation. No epileptic seizures were seen.

The patient had had two malignant diseases in the past: a seminoma (orchiectomy 1960) and a prostate carcinoma (interstitial radiotherapy 1994), but there were no signs of recurrence in recent years. In 1995 he was treated as an inpatient for myocardial infarction, from which he recovered well. The only regular medication consisted of aspirin and metoprolol. The patient gave up smoking in 1993 and his alcohol consumption was regular
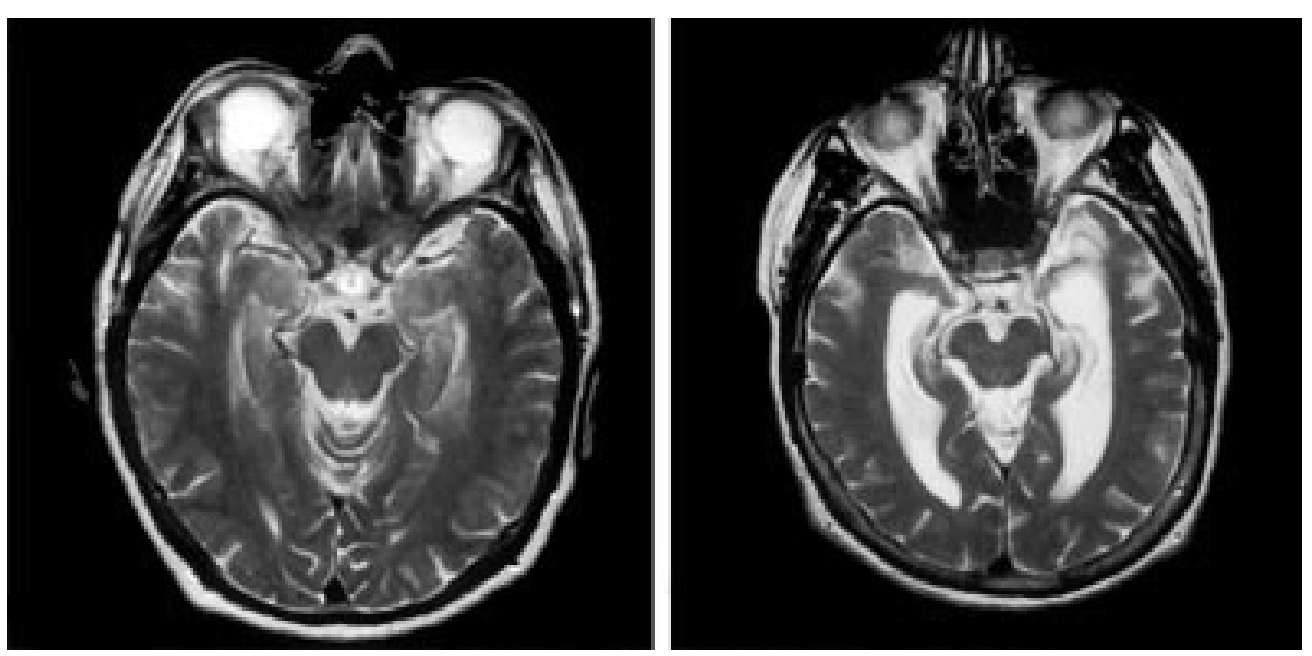

Figure 1 Comparison of axial T2 weighted MRI at the level of the midbrain in cases 1 and 2 showing a normal appearance in case 1 (left) compared with severe hippocampal atrophy in case 2 (right). but moderate. A chest radiograph performed in summer 1995 was normal as were routine blood tests. There was family history of cancer (mother died of stomach carcinoma) and heart disease (father and one of three brothers died of myocardial infarction).

On admission, the patient was severely disorientated and unable to retain any information for longer then a few minutes. Indeed, his memory loss was so profound that 5 minutes after the lumbar puncture he could not remember any procedure being performed on him! Apart from being considerably overweight, his general medical and neurological examination were normal. The patient was often distressed and painfully aware of his memory problems. He showed great concern for his wife, on whom he was entirely dependent in all practical matters.

\section{INVESTIGATIONS}

Routine blood tests were within the normal range and neuronal antibody screening, performed at Guy's Hospital, London, did not yield any positive results (in particular, the results for anti-Hu, $\mathrm{Ri}$, and Yo were all negative). Examination of CSF was performed twice. The first disclosed seven cells (six polymorphs and one lymphocyte) and normal protein $(0.4 \mathrm{~g} / \mathrm{l})$. On the second occasion there were seven lymphocytes and the protein concentration was increased $(0.7 \mathrm{~g} / \mathrm{l})$. Both examinations showed positive oligoclonal bands in CSF, but not in serum, suggesting intrathecal synthesis. The MRI with coronal T1 and axial T2 sequences was normal (figs 1 and 2 for comparison of cases). In particular, there were no abnormalities in the medial temporal lobes. An HMPAO-SPECT (fig 3) showed possible bilateral frontal and more clear cut, left sided temporal hypoperfusion. No pathological changes were seen on EEG and visual evoked potentials.

Repeated chest radiography raised the suspicion of a thoracic mass and a subsequent thorax and abdomen CT disclosed a tumour, arising in the right lower and involving the right middle lobe, about $4 \mathrm{~cm}$ in diameter. There 
were also nodal masses at the right hilum and in the subcarinal region. Bronchial biopsy confirmed a small cell carcinoma.

COGNITIVE ASSESSMENT

Formal neuropsychological testing confirmed the clinical impression of a severe but circumscribed amnestic syndrome. The doors and people test, ${ }^{25}$ Wechsler memory scale revised (WMS-R)-logical memory subtest, ${ }^{26}$ and Rivermead behavioural memory tests (RBMT)story recall subtests ${ }^{27}$ all showed severe deficits (table 1), particularly when compared with the expected premorbid levels. By contrast, performance on tests of remote memory, including the 50 item famous faces and famous events test of Greene and Hodges, ${ }^{28}$ was within the normal range (table 2). The discrepancy between remote and recent memory was particularly clear on the autobiographical memory interview (AMI), ${ }^{29}$ where the patient's performance was normal for childhood and early adult life, but clearly impaired for recent life (table 3).

Tests of working memory and frontalexecutive function showed normal performance including forward and backward digit span, verbal fluency for letter and semantic
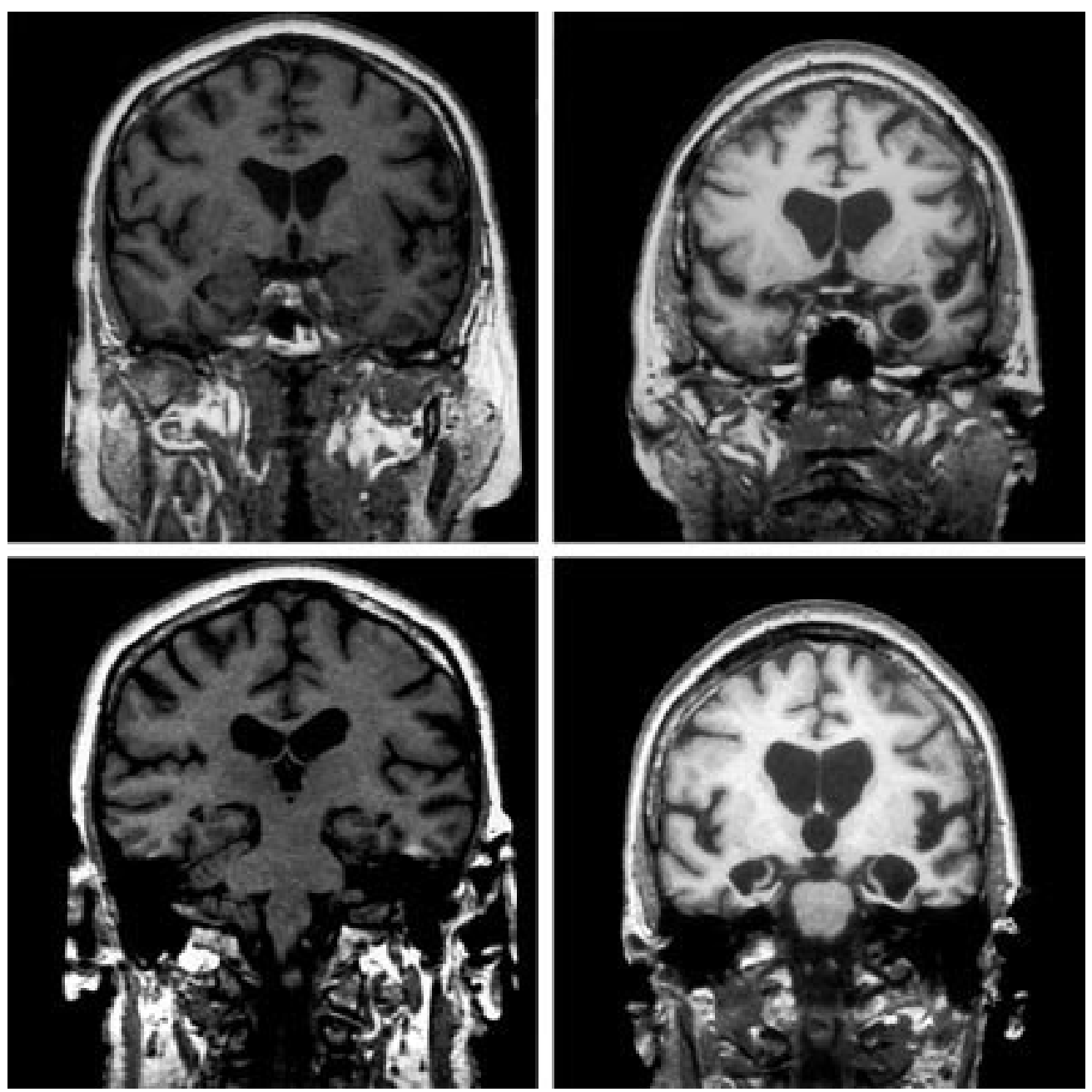

Figure 2 Comparison of coronal T1 weighted MRI images at the level of the amygdala (above) and hippocampus (below) in cases 1 (left) and 2 (right) showing a normal appearance in case 1 and severe, bilateral, but predominantly left sided atrophy in the amygdala and hippocampus in case 2.

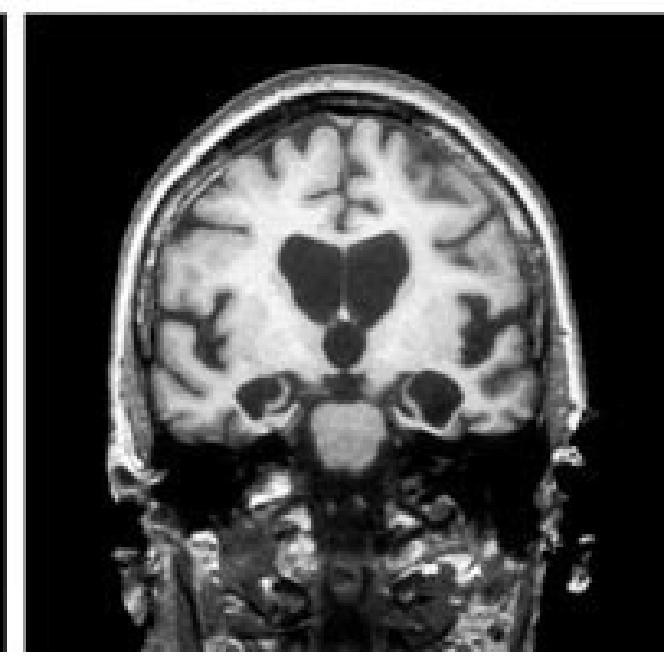

categories, and the modified Wisconsin card sorting test (WCST, ${ }^{30}$ table 4). The patient failed only on the six element test from the behavioural assessment of the dysexecutive syndrome (BADS) battery: ${ }^{31}$ a test in which subjects are required to plan and conduct six different tasks over 10 minutes, hence posing high demands on memory function during this period. No abnormalities were detected in his language function and his performance on the graded naming test ${ }^{32}$ and on WAIS-Rvocabulary subtests was normal (table 4 ). On all WAIS-R subtests his scores were either average (digit span, arithmetic, similarity judgement) or high average (vocabulary, block design, naming).

COURSE

Between February and April 1996 the patient underwent four cycles of chemotherapy (doxorubicin, cyclophosphamide, etoposide) followed by mediastinal radiotherapy between May and June (40 Gy in 15 fractions over 3 weeks). After the first two cycles of chemotherapy a progressive improvement in memory was noted. The patient was able to recount recent life events, including details about his chemotherapy, but remained amnesic for the 


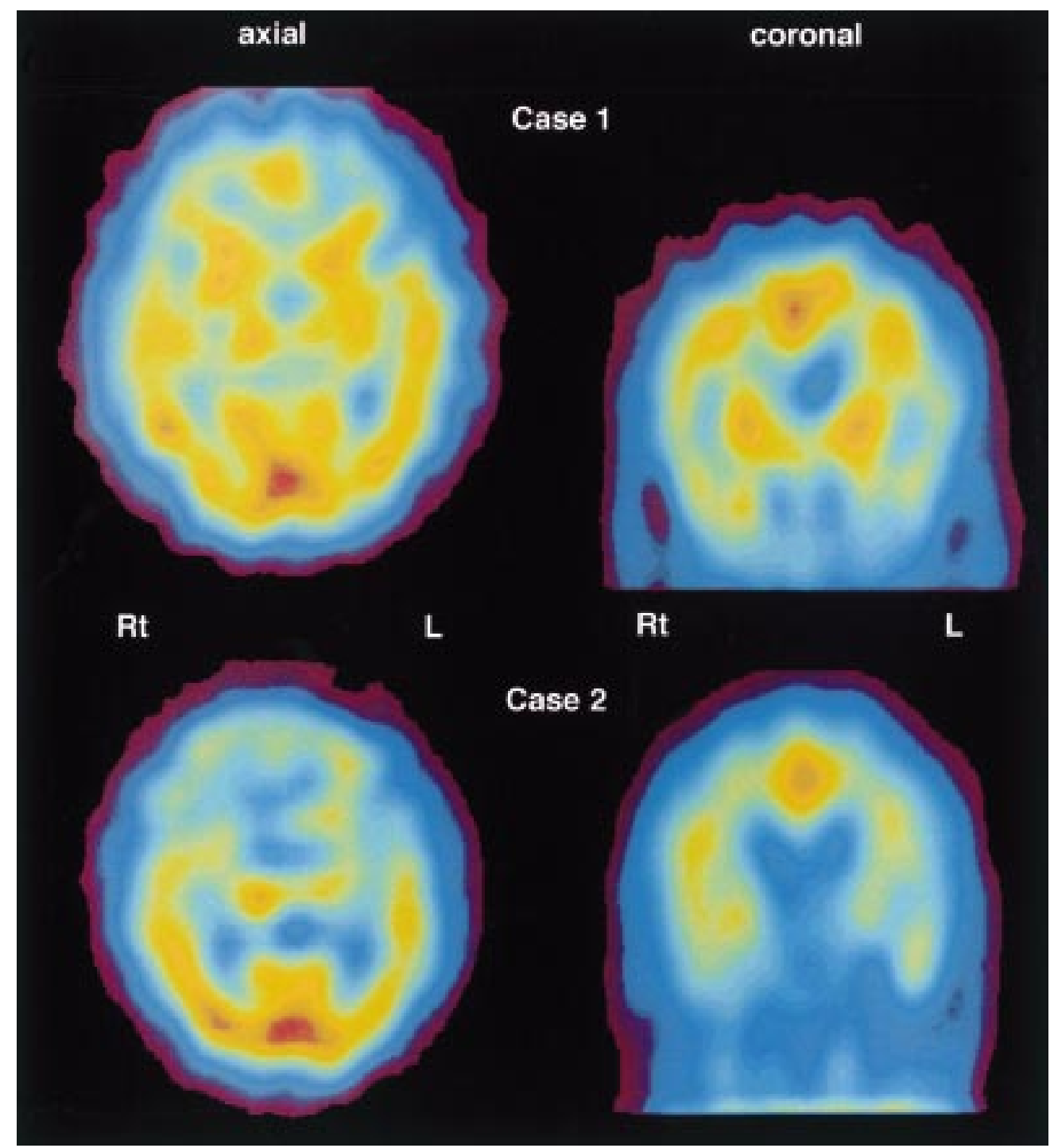

Figure 3 Comparison of HMPAO-SPECT in cases 1 and 2 showing mild, predominantly left sided hypoperfusion in case 1 and severe temporal and moderate frontal hypoperfusion in case 2.

Table 1 Results of standardised tests of anterograde memory in patients 1 and 2 before (the first examination) and after (the second and third examinations) treatment. All results are expressed in percentiles in comparison with normal, aged matched controls

\begin{tabular}{|c|c|c|c|c|c|c|}
\hline & \multicolumn{3}{|l|}{ Patient 1} & \multicolumn{3}{|c|}{ Patient 2} \\
\hline & $\begin{array}{l}\text { Febuary } \\
1996\end{array}$ & $\begin{array}{l}\text { Fune } \\
1996\end{array}$ & $\begin{array}{l}\text { April } \\
1997\end{array}$ & $\begin{array}{l}\text { April } \\
1998\end{array}$ & $\begin{array}{l}\text { April } \\
1999\end{array}$ & $\begin{array}{l}\text { Fuly } \\
1999\end{array}$ \\
\hline \multicolumn{7}{|l|}{ Doors and people test: } \\
\hline Immediate visual recall & 1 & 90 & 75 & 1 & 1 & 1 \\
\hline Delayed visual recall & 1 & 75 & 75 & 1 & 1 & 1 \\
\hline Visual recognition & 1 & 90 & 90 & 5 & 1 & 1 \\
\hline Immediate verbal recall & 1 & 25 & 75 & 1 & 1 & 1 \\
\hline Delayed verbal recall & 1 & 99 & 50 & 1 & 1 & 1 \\
\hline Verbal recognition & 10 & 50 & 10 & 5 & 5 & 5 \\
\hline \multicolumn{7}{|l|}{ WMS-R logical memory: } \\
\hline Immediate recall & 20 & 80 & 87 & 5 & 5 & 5 \\
\hline Delayed recall & 1 & 75 & 94 & 1 & 1 & 1 \\
\hline
\end{tabular}

Table 2 Performance on famous event and famous faces test

\begin{tabular}{llll}
\hline & Patient 1 & Patient 2 & Controls $(n=30)$ \\
\hline Famous events test: & 48 & 34 & $41.0(7.4)$ \\
$\quad$ Correctly recognised as real (50) & 39 & 12 & $38.2(6.6)$ \\
$\quad$ Hits minus false alarms (50) & 36 & 0 & $31.1(9.0)$ \\
Famous faces test: & 38 & 0 & $39.3(8.8)$ \\
$\quad$ Named (50) & & & \\
$\quad$ Identified (50) &
\end{tabular}

Maximum scores for each subtest are given in parentheses.

Values for controls in parentheses are SD. period December 1995 to March 1996. The regression of the tumour was confirmed by thoracic CT, the recovery of memory by neuropsychological testing. A repeated doors and people test showed a superior visual and an average verbal performance, well in keeping with the patient's own estimation of the degree of his recovery (table 1). Interestingly, verbal recognition, least affected during the disease, remained the only subtest on which the patient's performance was under average. His condition remained stable over the next year and the patient undertook several professional

Table 3 Performance on the autobiographical memory interview (AMI) by contast with published cut off values

\begin{tabular}{llll}
\hline & Patient 1 & Patient 2 & Cut off \\
\hline Incidents: & & & \\
$\quad$ Childhood (9) & 9 & 6 & 5 \\
$\quad$ Early adult life (9) & 8 & 2 & 6 \\
$\quad$ Recent life (9) & 3 & 0 & 6 \\
Personal facts: & & & \\
$\quad$ Childhood (21) & 21 & 15 & 14 \\
$\quad$ Early adult life (21) & 21 & 13 & 16 \\
$\quad$ Recent life (21) & 13 & 3 & 18 \\
\hline
\end{tabular}

Maximum scores for each subtest are given in parentheses. 
Table 4 Tests of frontal-executive functions, semantic knowledge and language (maximum scores in parentheses)

\begin{tabular}{lcc}
\hline Test & Patient 1 & Patient 2 \\
\hline Digit span: & 7 & 6 \\
$\quad$ Forwards & 5 & 7 \\
$\quad$ Backwards & 64 & 33 \\
Verbal fluency-letters (FAS in 180 s): & 5 & 8 \\
$\quad$ Correct answers & & \\
$\quad$ Perseverations & 22 & 5 \\
Verbal fluency-category (animals in 60 s): & 5 & 4 \\
$\quad$ Correct answers & 6 & $14(10)$ \\
$\quad$ Perseverations & $6(1)$ & 18 \\
Wisconsin card sorting test (WCST): & 14 & 14 \\
$\quad$ Achieved categories (6) & 12 & 51 \\
$\quad$ Number of errors (perseverations) & 24 & 48 \\
WAIS-R-similarities (28) & 50 & 78 \\
WAIS-R vocabulary age scaled & NT & \\
Graded naming test (30) & NT & \\
Pyramids and palm trees (52) & & \\
Noun and verb synonym (48) & & \\
Test for the reception of grammar (80) &
\end{tabular}

Maximum scores for each test are given in parentheses.

NT $=$ Not tested.

journeys overseas, where his expertise on damage estimation after natural disasters was highly valued. Regular oncological and neuropsychological checks showed no signs of recurrence.

In January 1998, more than 2 years after initial presentation, the patient was admitted as an emergency to hospital with a 1 week history of progressive dyspnoea. Shortly after admission he had a cardiac arrest from which resuscitation was unsuccessful. The postmortem examination confirmed myocardial ischaemia leading to acute left ventricular failure as the cause of death. There was no evidence of recurrence of any of the previously diagnosed malignancies: prostatic, testicular, and small cell lung carcinoma. Macroscopical examination of the brain and meninges was unremarkable. Unfortunately, neurological history was not made known to the pathology department and microscopic examination of the brain was not conducted.

\section{Case 2}

PRESENTATION

A 69 year old right handed female university graduate and former A level examiner in English and French literature, was admitted to Addenbrooke's Hospital, Cambridge with several months history of persistent cough and intermittent chest pain and fever and a 2 week history of sudden onset memory loss. She was generally fit and previously led an active life. The medical history consisted only of hypertension, well controlled with bendrofluazide, and a right sided total hip replacement in February 1997. She had never smoked and her alcohol consumption was moderate. The family history included neoplasms in her father and older brother and a ruptured intracerebral aneurysm leading to death from subarachnoid haemorrhage in her twin sister. Diseases associated with cognitive dysfunction and psychiatric disorders had not been reported.

Repeated chest radiography in summer and autumn 1997 were entirely normal, as was the physical examination and ECG. Blood tests showed a slight rise in ALT, erythrocyte sedimentation rate, and $\mathrm{C}$ reactive protein. Transthoracic and transoesophageal echo- cardiography were normal and the patient's condition stabilised in the first months of 1998 . Then, in March 1998, she appeared suddenly disoriented in her own house, started asking repetitive questions, instantly forgetting the answers, and was unable to recall any recent events. Her condition resembled transient global amnesia but showed no signs of recovery. She was, therefore, admitted to hospital.

On the ward she was completely disoriented in place and time. At times she thought she was in a hospital, at other times in a hotel. She did not admit to a memory problem and explained her inability to answer questions stating that "I have just arrived at this new place after a long flight and I am still jet lagged". On rainy days she tended to think that she was in Canada, on fair days in Australia (she knew both countries from visits to her relatives). Subsequent assessment of her remote memory showed that she was able to recall the key events of her early life up to graduating from Cambridge aged 22, but could produce no distinct episode from the past 30 years or so and only very scarce factual details. Her general physical and neurological examinations, however, remained normal throughout her stay in hospital.

\section{INVESTIGATIONS}

As on former occasions, routine laboratory tests did not show any abnormalities. Repeated chest radiography suggested widened mediastinum, subsequent chest CT showed mediastinal adenopathy, and mediastinoscopy confirmed the diagnosis of small cell carcinoma. An octreotide scintigram did not show any evidence of abnormal intake in the body or skull suggestive of metastatic disease. The antibody screening performed at Guy's Hospital, London, including anti-neuronal $(\mathrm{Hu} / \mathrm{Ri})$ and antiPurkinje cell (Yo) antibodies, was negative.

The initial CT, performed in March 1998, before the beginning of treatment, was normal. Six months later repeat CT showed clear cut medial temporal atrophy (fig 4). The three dimensional MRI, performed after the completion of treatment in November 1998, also showed dramatic changes: as illustrated in figs 1 and 2 there was severe atrophy of the medial temporal lobes with an almost complete loss of the hippocampus and the parahippocampal gyrus (including the entorhinal and perirhinal cortex) bilaterally and the amygdala complex, especially on the left. There was also a less pronounced atrophy of the thalamus and a mild degree of generalised cortical loss. The HMPAO-SPECT showed markedly decreased perfusion in frontal and medial temporal lobes bilaterally (fig 3 ).

\section{COGNITIVE ASSESSMENT}

The most pronounced deficit on neuropsychological testing was an almost complete loss of anterograde memory: the immediate recall of a story was minimal (2/47 on WMS-R logical memory, $1 / 21$ on RBMT story), the delayed recall invariably zero. On the doors and people test the severe impairment affected all subtests, with practically non-existent verbal $(0 / 36)$ and visual (5/36) recall and recognition barely 

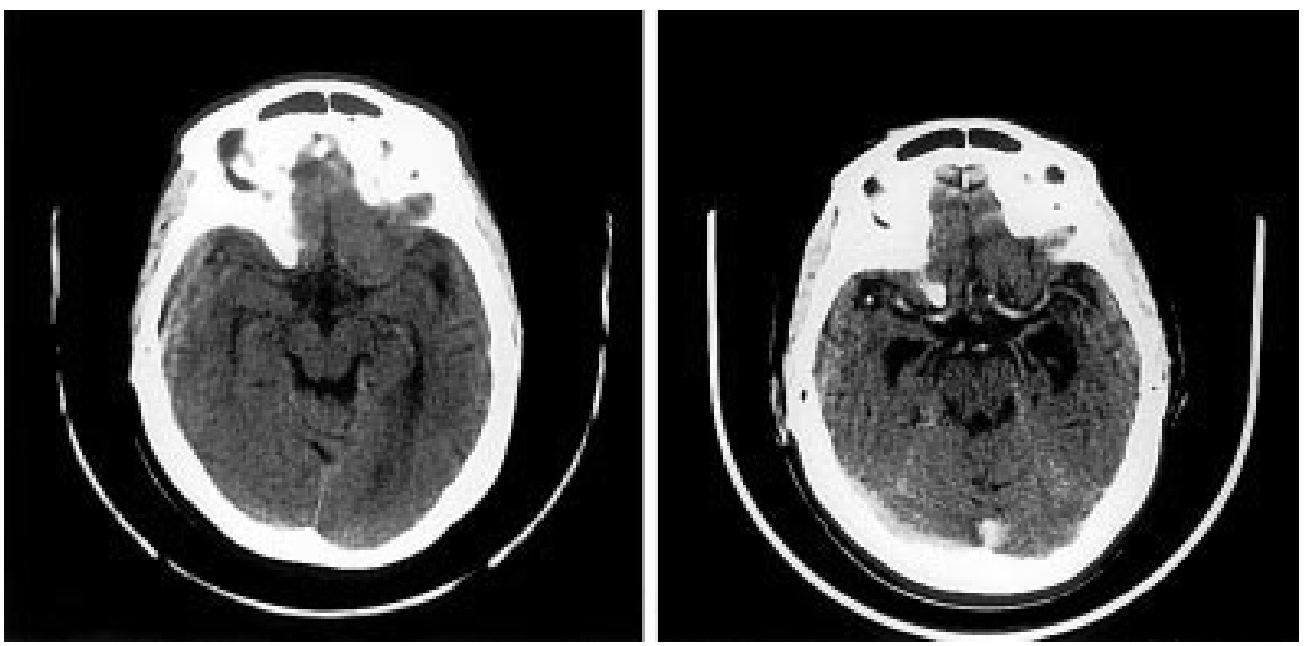

Figure 4 Comparison of two consecutive CT scans in case 2, first before treatment in March 1998 (left), and after completion of chemotherapy in August 1998 (right). A normal, age related appearance on the first scan contrasts with marked atrophy and ventricular dilatation on the second.

exceeding the chance level (table 1). On the Rey auditory verbal learning test the patient was only able to remember the three most recent items out of a list of 15 after the fifth reading, and was entirely at chance at recognising them out of a list of 50 . The deficits extended also to remote autobiographical and semantic memory.

Knowledge of past events and people was severely degraded (table 2). On the 50 item famous events test she identified as real 34 of the 50 true events, but also endorsed a very large number of foils giving her a very poor $\mathrm{d}^{1}$ score of 12 (hits minus false alarms). On the famous faces test, she failed to name any of the faces and could not produce any accurate identifying information. Results of the AMI showed normal performance for section A (childhood) only, with subsequent increasing impairment for early adult and particularly recent life, where she was scarcely able to produce any specific information (table 3 ).

The cognitive deficits were, however, not confined to memory. The patient was severely anomic: she was able to name only one out of 30 items on the graded naming test (table 4), although she could produce appropriate semantic information for 17 of them. In a few instances she also produced inappropriate semantic information-for example, calling a kangaroo "an African animal". By contrast, her performance on tests of non-verbal semantic knowledge (pyramids and palm trees, ${ }^{33}$ noun and verb synonyms ( $R$ Berndt, personal communication) and syntactic comprehension (test for the reception of grammar TROG), ${ }^{34}$ were within the normal range (table 4). Despite the anomia she was also displaying remarkable eloquence in the verbal WAIS subtests "similarities" and "vocabulary" (table 4).

Even more striking than the general anomia was her inability to produce proper names: in the first interviews the only famous names she was able to recall were "Molière" and "Dickens". The anomia for proper names was most clearly evident on a test of recognition of 30 very famous faces. ${ }^{35}$ She was able to recognise 26 as "famous" (rejecting correctly eight out of
10 foils), but she could only give specific information for seven and was unable to name a single one.

On frontal-executive tests her performance on verbal fluency was low average ( $11 /$ minute for letters, 10/minute for categories with occasional perseverations) and on the WCST she could obtain four categories producing 10 perseverations (table 4). The six elements test could not be completed due to high distractibility. With the exception of the "silhouettes" subtest (requiring naming and hence sensitive to anomia) she passed without any difficulty all other subtests of the visual object and space perception battery, ${ }^{36}$ indicating unimpaired visuospatial processing.

COURSE

Between April and July 1998 the patient completed four courses of chemotherapy, consisting of a combination of doxorubicin, etoposide and cyclophosphamide. On the CT of the thorax there were no signs of tumour growth, but no regression of the mediastinal lymphadenopathy. In view of this the decision was made not to recommend any further mediastinal radiotherapy.

No change, however, has been noticed in the cognitive state of the patient. Her behaviour remained the same, with repetitive questioning, immediate forgetting and only very limited insight. Repeated tests of memory showed the same degree of impairment as before the initiation of treatment (table 1). Interestingly, the patient is still able to play bridge and chess relatively well, although at times she fails to recognise her partners, with whom she used to play for many years.

\section{Discussion}

The association between limbic encephalitis and carcinoma, as postulated by Corsellis et $a l^{2}$ has been confirmed by many case reports describing patients with an acute or subacute onset of amnesia and other cognitive deficits, followed weeks to months later by diagnosis of a neoplasm. ${ }^{3-10}$ 14-16 Both patients presented here showed several characteristic features 
often associated with the diagnosis of PNLE. The pattern of clinical presentation was characterised by memory loss, repetitive questioning, and disorientation in time and place, even if the acuteness of onset was somewhat unusual. The diagnosis of the small cell lung cancer followed the appearance of cognitive abnormalities by several weeks. In both patients, severe anterograde amnesia constituted the core of the neuropsychological deficit. Detailed neuropsychological testing showed impairment of both active recall and passive recognition, which cannot be explained by a pure difficulty in accessing the contents of memory. In both cases there was an impressive discrepancy between the inability to retain new information over time and the intact working memory (as measured by the superior results on forward and backward digit span). Subtle deficits were found on frontal-executive tests. The first patient's difficulty on the six elements test might be explicable in terms of the high amnestic demands of this task. The more pronounced frontal deficits in the second patient, on tests such as WCST and verbal fluency, could be partly accounted for by a combination of impaired memory (also explaining the perseverations) and severe anomia. It is not certain to what extent the frontal dysfunction might also be contributing to the anosognosia (lack of awareness of symptoms) in the second patient. There was no impairment of visuospatial processing in either patient.

Although cases of limbic encephalitis with complete remission have been reported before,${ }^{18-20}$ improvement has come from clinical observations rather than documentation by neuropsychological testing. Our patient (case 1) is the first to be substantiated by detailed neuropsychological evaluation.

Several contrasting features in the two patients are of particular interest. Firstly, patient 1 with profound anterograde amnesia had normal MRI yet abnormal HMPAOSPECT imaging. This dissociation between normal structural and abnormal functional brain imaging has been previously described in PNLE: two recent studies have described increased metabolic activity in the medial temporal region in FDG-PET which the authors have argued reflects acute inflammatory change in the medial temporal lobe. ${ }^{12}{ }^{13} \mathrm{Al}-$ though patient 1 showed changes on HMPAOSPECT confined to the left side, we suspect that more sensitive methods would have shown bilateral pathology as global amnesia of this type is almost invariably associated with bilateral disruption of the temporolimbic circuits. ${ }^{37}{ }^{38}$ It should be noted, however, that persistent amnesia after left unilateral strokes involving medial temporal or thalamic regions have been reported (for review see Ott and Saver $\left.{ }^{39}\right)$. By contrast with patient 1 with full recovery, patient 2 had severe bilateral amygdalar and hippocampal atrophy which developed over a period of 6 months.

The relation between anterograde and remote autobiographical memory remains controversial. The neuroimaging and pathological findings in patients with extensive autobio- graphical amnesia are diverse: according to some authors the hippocampal region has a critical role in the long term storage and representation of episodic memory throughout one's lifespan. ${ }^{40}$ By contrast, others favour the idea that such memories have distributed (hippocampally independent) cortical representations so that diffuse pathology, as seen in Alzheimer's disease or in association with multifocal vascular disease has a particular effect on such memories. ${ }^{41}$ Still others claim a particular role for right frontotemporal regions in autobiographical memory. ${ }^{42}$ The finding of profound anterograde yet very limited retrograde amnesia in patient 1 clearly points to a dissociation in the processes and structures underlying these aspects of memory. The extensive retrograde amnesia of the second patient was paralleled by more widespread structural and functional damage, including the hippocampal complex, entorhinal and perirhinal cortices, and the amygdala, as documented by the MRI and SPECT.

Another impressive difference between our patients was the performance on naming tasks: whereas the first patient's results were normal, the second patient was severely anomic, particularly considering her high premorbid level of functioning. The inappropriate semantic information produced by the patient when asked to describe some of the items in the graded naming test might indicate a subtle semantic deficit, but her performance was normal on other more specific visual and verbal semantic tests, which do not require naming (for example, pyramids and palm trees, noun and verb synonym judgement tests) suggesting a largely postsemantic cause for her anomia.

It is difficult to explain the dramatic differences in presentation and course between our patients. One possible explanation could be seen in a quantitatively different response to chemotherapy: a complete remission of the tumour in the first patient, but only a partial one in the second. This effect cannot be due to a delay in treatment, as it was started in the second patient several weeks earlier after the onset of symptoms than in the first one. In addition, consecutive brain CT demonstrated the progression of atrophy despite the remission of the bronchial tumour. Another hypothesis relates to differences in the type of antibodies involved..$^{15}$ Alamawitch et $a l^{15}$ compared eight patients with positive, and eight with negative, anti-Hu antibodies, all with a clinical history suggestive of PNLE and histologically confirmed small cell lung cancer. By contrast with those with positive antibody results, the anti-Hu negative patients had more isolated limbic involvement and a higher rate of recovery after treatment. Both of our patients were anti-Hu antibody-negative. It seems likely that, as yet unknown, antibodies might be involved in the second patient, particularly as new antibodies are currently being identified in various paraneoplastic syndromes. ${ }^{43}$ Ultimately, only a combination of detailed cognitive assessment, neuroimaging, immunological screening, and postmortem histology will illuminate the nature of PNLE and explain its 
clinical heterogeneity. These issues are of considerable scientific and therapeutic importance in view of a possible recovery, as illustrated by the radical recovery seen in our first patient. In conclusion, we suggest that cognitive deficits beyond anterograde amnesia and evidence of destructive medial temporal pathology on neuroimaging are both poor prognostic features in PNLE.

We thank I M S Wilkinson for referral of the first patient. We are indebted to the late Kristin Breen and to Jon Evans for supply-
ing additional data on case 1 . THB and JRH are supported by ing addition

1 Brierly JB, Corsellis JAN, Hierons R, et al. Subacute encephalitis of late adult life mainly affecting the limbic encephalitis of late adult life
areas. Brain 1960;83:357-68.

2 Corsellis JA, Goldberg GJ, Norton AR. "Limbic encephalitis" and its association with carcinoma. Brain 1968;91:48196.

3 Tsukamoto T, Mochizuki R, Mochizuki H, et al. Paraneoplastic cerebellar degeneration and limbic encephalitis in patient with adenocarcinoma of the colon. I Neurol Neurosurg Psychiatry 1993;56:713-16.

4 Bakheit AMO, Kennedy PGE, Behan PO. Paraneoplastic limbic encephalitis: clinico-pathological correlations. $f$ Neurol Neurosurg Psychiatry 1990;53:1084-8.

5 Antoine JC, Honnorat J, Anterion CT, et al. Limbic encephalitis and immunological perturbations in two patients with thymoma. $\mathcal{F}$ Neurol Neurosurg Psychiatry 1995;58:706-10.

6 Sutton RC, Lipper MH, Brashear HR. Limbic encephalitis occurring in association with Alzheimer's disease. $¥$ Neurol occurring in association with Alzheim

7 Vollmer J, Ferbert A, Thron A. Verlaufsbeobachtung bei pollmer J, Ferbert A, Thron A. Verlaufsbeobachtung bei paraneoplastischer

8 Kodama T, Numaguchi Y, Gellad FE, et al. Magnetic resonance imaging of limbic encephalitis. Neuroradiol 1991;33 520-3

9 Deodhare S, O'Connor P, Ghazarian D, et al. Paraneoplastic limbic encephalitis in Hodgkin's disease. Can $\mathcal{F}$ Neuro Sci 1996;23:138-40.

10 Rimmelin A, Sellal F, Morand G, et al. Imaging of limbic paraneoplastic encephalitis. F Radiol 1997;78:73-6.

11 Lacomis D, Khoshbin S, Schick RM. MR imaging of paraneoplastic limbic encephalitis. F Comput Assist Tomogr 1990;14:115-7.

12 Provenzale JM, Barboriak DP, Coleman RE. Limbic encephalitis: comparison of FDG PET and MR imaging encephalitis: comparison of FDG PET

13 Fakhoury T, Abou-Khalil B, Kessler RM. Limbic encephalitis and hyperactive foci on PET scan. Seizure 1999;8:427litis and

14 Dalmau J, Graus F, Rosenblum MD, et al. Anti-Hu associated paraneoplastic encephalomyelitis/sensory neuronopathy: a clinical study of 71 patients. Medicine 1992;71:59-72.

15 Alamowitch S, Graus F, Uchuya M, et al. Limbic encephalitis and small cell lung cancer: clinical and immunologica features. Brain 1997;120:923-8.

16 Touze E, Auliac JB, Carras P, et al. Limbic encephalitis and SIADH revealing small-cell anaplastic lung cancer: MR and immunologic findings. Rev Neurol (Paris) 1998;154: 539-41.

17 Carr I. The Ophelia syndrome: memory loss in Hodgkin's disease. Lancet 1982;i:844.
18 Kaniecki R, Morris JC. Reversible paraneoplastic limbic encephalitis. Neurology 1993;43:2418-19.

19 Nokura K, Yamamoto H, Okawara Y, et al. Reversible limbic encephalitis caused by ovarian teratoma. Acta Neurol Scand 1997;95:367-73.

20 Burton GV, Bullard DE, Walther PJ, et al. Paraneoplastic limbic encephalopathy with testicular carcinoma. Cancer 1988;62:2248-51.

21 Ahern G, O'Connor M, Dalmau J, et al. Paraneoplastic temporal lobe epilepsy with testicular neoplasm and atypical amnesia. Neurology 1994;44:1270-4.

22 Martin RC, Haut MW, Goeta-Kreisler K, et al. Neuropsychological functioning in a patient with paraneoplastic limbic encephalitis. F Int Neuropsychol Soc 1996;2:460-6.

23 Hodges JR. Transient amnesia: clinical and neuropsychological aspects. London: Saunders JB, 1991.

24 Hodges JR. Unravelling the enigma of transient global amnesia. Ann Neurol 1998;43:151-3.

25 Baddeley AD, Emslie H, Nimmo-Smith I. The doors and people test: a test of visual and verbal recall and recognition. Bury St Edmunds, Suffolk: Thames Valley Test Company, 1994.

26 Wechsler D. Wechsler memory scale - revised (WMS-R). San Antonio: The Psychological Corporation, 1974.

27 Wilson BA, Cockburn J, Baddeley AD. The Rivermead behavioural memory test. Reading: Thames Valley Test Company, 1985.

28 Greene JDW, Hodges JR. Identification of famous faces and names in early Alzheimer's disease: relationship to anterograde episodic and semantic memory impairment. Brain 1996;119:111-28.

29 Kopelman MD, Wilson BA, Baddeley AD. The autobiographical memory interview. Bury St Edmunds, Suffolk: Thames Valley Test Company, 1990.

30 Nelson HE. A modified card sorting test sensitive to frontal lobe defects. Cortex 1976;12:313-24.

31 Wilson BA, Alderman N, Burgess PW, et al. Behavioural assessment of the dysexecutive syndrome. (BADS). Bury St Edmunds, Suffolk: Thames Valley Test Company, 1996.

32 McKenna P, Warrington EK. Graded naming test. Windsor: NFER-Nelson, 1983.

33 Hanley JR, Young AW, Pearson NA. Defective recognition of familiar people. Cogn Neuropsychol 1989;6:179-210.

34 Howard D, Patterson K. Pyramids and palm trees: a test of semantic access from pictures and words. Bury St Edmunds, Suffolk: Thames Valley Test Company, 1992.

35 Bishop DVM. Test for the reception of grammar. Cambridge: Medical Research Council, 1989

36 Warrington EK, James $M$. The visual object and space perception battery. Bury St Edmunds, Suffolk: Thames Valley Test Company, 1991.

37 Squire LR. Memory and the hippocampus: a synthesis from findings with rats, monkeys, and humans. Psychol Rev 1992;99:195-231.

38 Vargha-Khadem F, Gadian DG, Watkins KE, et al. Differential effects of early hippocampal pathology on episodic and semantic memory. Science 1997;277:376-80.

39 Ott BR, Saver JL. Unilateral amnesic stroke: six new cases and a review of the literature. Stroke 1993;24:1033-42.

40 Nadel L, Moscovitch M. Memory consolidation, retrograde amnesia and the hippocampal complex. Curr Opin Neurobiol 1997;7:217-27.

41 Evans J, Breen E, Antoun N, et al. Focal retrograde amnesia for autobiographical events following cerebral vasculitis: a connectionist account. Neurocase 1996;2:1-12.

42 Kroll NEA, Markowitsch HJ, Knight RT, et al. Retrieval of old memories: the temporofrontal hypothesis. Brain 1997;120:1377-99.

43 Dalmau J, Gultekin HS, Posner JB. Paraneoplastic neurologic syndromes: pathogenesis and physiopathology. Brain Pathol 1999;9:275-84. 